\title{
Job strain predicts recurrent events after a first acute myocardial infarction: The Stockholm Heart Epidemiology Program
}

\section{Lo stress da lavoro predice le recidive dopo un primo infarto miocardico: The Stockholm Heart Epidemiology Program}

\author{
Commento bibliografico di Domenico Del Forno e Valentina Battimiello
}

Monaldi Arch Chest Dis 2010; 74: 47-48. Dipartimento di Medicina Clinica, Scienze Cardiovascolari ed Immunologiche - Settore Intervento Psicologico in Riabilitazione
Cardiologica - Università degli Studi di Napoli Federico II.

Làszlo e coll [1] presentano, nel loro articolo "Job strain predicts recurrent events after a first acute myocardial infarction: The Stockholm Heart Epidemiology", recentemente pubblicato su Journal of Internal Medicine, i risultati di uno studio prospettico effettuato nel tentativo di chiarire l'associazione tra stress lavoro-correlato ed eventi cardiovascolari dopo un infarto miocardico (IMA). In effetti, se esiste in letteratura un certo grado di accordo sul fatto che lo stress sul lavoro aumenta il rischio di sviluppare malattie cardiovascolari, poco chiaro appare invece il ruolo prognostico che questo riveste rispetto alla possibilità che si verifichino eventi cardiaci sfavorevoli una volta occorso il primo infarto. Inoltre, nonostante il fatto che le malattie cardiovascolari rappresentino nel nostro paese la prima causa di morte e la principale causa di malattia ed invalidità, e a dispetto della riconosciuta influenza che lo stress lavoro-correlato ha sulla salute individuale, si possono considerare ancora insufficienti gli studi che si sono occupati di chiarire i meccanismi responsabili di tale associazione.

In sintesi, lo scopo del lavoro è duplice: da una parte, verificare se lo stress sul lavoro predice recidive di eventi cardiovascolari dopo il primo infarto miocardico e, dall'altra, analizzare la validità e attendibilità delle teorie più note che spiegano la relazione tra stress cronico sul lavoro e malattie cardiovascolari. Tali teorie fanno riferimento a due possibili meccanismi, uno diretto ed un altro indiretto. Il primo meccanismo implica che lo stress, attraverso l'alterazione funzionale del sistema nervoso autonomico e dell' asse ipotalamico-pituitario-adrenalinico, provoca cambiamenti metabolici, infiammatori ed omeostatici che aumentano il rischio di eventi car- diaci negativi; il secondo evidenzia che lo stress lavoro-correlato influenza la salute indirettamente, inducendo l'acquisizione di uno stile di vita non salutare, caratterizzato dalla presenza di quelle abitudini ampiamente riconosciute come fattori di rischio per le malattie cardiovascolari: il fumo di sigaretta, la mancanza di una dieta adeguata, l'assenza di esercizio fisico ed il consumo di alcool.

Gli autori hanno scelto di seguire soggetti riconosciuti come casi di IMA non fatale all'interno del Stockholm Heart Epidemiology Program (SHEEP). Più nello specifico, hanno condotto la loro ricerca su 676 soggetti, sia maschi che femmine, d'età compresa tra i 45 e i 65 anni, con un lavoro retribuito al momento del loro infarto. Su di essi hanno raccolto tre tipi di dati, fondamentali ai fini dello studio:

- Informazioni relative a fattori demografici, legati al lavoro, clinici e legati allo stile di vita, attraverso la somministrazione di un questionario durante il periodo di ospedalizzazione;

- Informazioni riguardanti lo stress lavoro-correlato, attraverso la somministrazione dello Swedish Demand-Control Questionnaire. Tale questionario misura lo stress attraverso due scale: la scala delle Richieste Psicologiche, che verifica se le caratteristiche del lavoro implicano il dover lavorare a ritmi elevati, duramente e con molto sforzo, con richieste contrastanti e poco tempo per portare a termine i compiti; e la scala del Controllo Lavorativo, che verifica se le persone hanno la possibilità di decidere come portare avanti il proprio lavoro e se l'attività svolta offre la possibilità di imparare nuove abilità, richiede alti livelli di competenze acquisite e di creatività. Sulla base di queste due scale gli autori 
hanno diviso i soggetti in quattro gruppi: 1) i pazienti con basse richieste lavorative e un alto livello di controllo hanno costituito il gruppo "a basso stress"; 2) quelli con alte richieste e un alto livello di controllo hanno formato "gruppo attivo"; 3) i pazienti con basse richieste e bassi livelli di controllo hanno costituito il "gruppo passivo"; 4) infine, quelli con alte richieste e un basso livello di controllo sono stati classificati come il gruppo "ad alto stress".

- Informazioni biomediche per la valutazione del bilancio metabolico, infiammatorio ed omeostatico dell'organismo.

I risultati ottenuti attraverso l'analisi statistica dei dati raccolti mostrano come i soggetti che hanno avuto durante il periodo di follow-up nuovi infarti, fatali e non, sono principalmente raccolti nel gruppo ad alto stress, seguiti progressivamente da quelli che fanno parte del gruppo passivo, attivo e a basso stress.

In particolare, nel corso degli 8.5 anni di follow up, vi furono 155 casi di morte cardiaca o di IMA non fatale. In totale vi furono 96 casi di morte, delle quali 52 di origine cardiaca. Dopo aggiustamento per potenziali fattori confondenti, i pazienti con alto stress da lavoro avevano un rischio aumentato di morte cardiaca o IMA non fatale rispetto a quelli con basso stress da lavoro, con un Hazard ratio (HR) di 1.73 (96\% CI 1.06-2.83). Gli stessi risultati si ottenevano per la mortalità cardiaca (HR $2.81,95 \%$ CI 1.16-6.82) e per la mortalità totale (HR 1.65, 95\% CI 0.91-2.98). Pertanto, in sintesi, tali risultati indi- cano che esiste un'associazione tra stress e eventi cardiovascolari ricorrenti.

Allo stesso tempo, però, gli autori non hanno riscontrato nessuna evidenza che alterazioni del bilancio metabolico, infiammatorio, emocoagulativo ed omeostatico dell'organismo, o uno stile di vita a rischio influenzino significativamente la correlazione tra eventi cardiaci ricorrenti ed alti livelli di stresslavoro correlato. In altre parole, il potere prognostico dello stress esperito in ambito lavorativo in relazione ad eventi cardiovascolari ricorrenti non viene spiegato sulla base di quei meccanismi che vengono riconosciuti come i mediatori della relazione esistente tra questi due fattori.

La ricerca presentata si dimostra interessante per due motivi sostanziali: il primo, di ordine pratico, evidenzia come interventi mirati allo scopo di ridurre lo stress lavoro-correlato debbano acquisire un ruolo di primaria importanza nel campo della prevenzione delle patologie e delle recidive cardiovascolari; il secondo, di interesse teorico, mostra il lavoro che ancora deve essere svolto per aumentare le conoscenze in questo settore, che attualmente risultano, totalmente o in parte, insufficienti.

\section{Reference}

1. Laszlo KD, Ahnve S, Hallqvist J, et al. Job strain predicts recurrent events after a first acute myocardial infarction: The Stockholm Heart Epidemiology. J. Internal Medicine 2010; doi: 10.1111/j.1365-2796.2009.02196.x 